\title{
A New Stapes-Head Coupler for the Vibrant Soundbridge System
}

\author{
Birthe Warnholtz ${ }^{a, b}$ Merlin Schär ${ }^{a, b}$ Pascale Cuny ${ }^{a, b}$ Kathrin Sonntag ${ }^{c}$

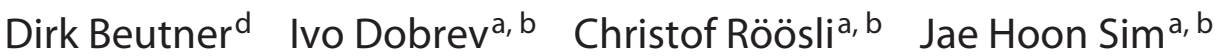 \\ aDepartment of Otolaryngology, Head and Neck Surgery, University Hospital Zürich, Zürich, Switzerland; \\ ${ }^{b}$ Department of Otolaryngology, Head and Neck Surgery, University of Zürich, Zürich, Switzerland; \\ 'MED-EL Medical Electronics, Innsbruck, Austria; 'Department of Otorhinolaryngology, Head and Neck Surgery, \\ University of Göttingen, Göttingen, Germany
}

\section{Keywords}

Stapes · Stapes-head coupler · Vibrant Soundbridge ·

Vibroplasty Clip coupler

\begin{abstract}
Introduction: The Vibrant Soundbridge (MED-EL Medical Electronics, Austria) is an active middle ear implant with a floating mass transducer (FMT) for patients with conductive, sensorineural, or mixed hearing loss. While the FMT is vertically aligned above the stapes head $(\mathrm{SH})$ with the current Vibroplasty Clip coupler (MED-EL Medical Electronics), the new SH coupler was developed to mount the FMT on the inferior side of the stapes and to fit in the reduced middle ear space after canal-wall-down mastoidectomy. Methods: Using 11 human cadaveric temporal bones (TBs), placements of the new $\mathrm{SH}$ couplers on the stapes were examined, and effective stimuli to the cochlea were evaluated by measuring piston-like motion of the stapes footplate with a current of $1 \mathrm{~mA}$ on the FMT. The results were assessed in comparison with the Vibroplasty Clip coupler. Results: The new SH coupler showed perfect coupling on the stapes in 9 out of 11 TBs. A small gap between the $\mathrm{SH}$ and the plate of the connection
\end{abstract}

karger@karger.com www.karger.com/aud

Karger $\stackrel{\text { ' }}{5}$

GOPEN ACCESS
(C) 2021 The Author(s)

Published by S. Karger AG, Basel

This is an Open Access article licensed under the Creative Commons Attribution-NonCommercial-4.0 International License (CC BY-NC) (http://www.karger.com/Services/OpenAccessLicense), applicable to the online version of the article only. Usage and distribution for commercial purposes requires written permission. link part was unavoidable in 2 TBs but had negligible effect on vibrational motion of the stapes. Vibrational motion of the stapes with the new $\mathrm{SH}$ coupler was reduced at frequencies above $3 \mathrm{kHz}$ compared to the corresponding motion with the current Vibroplasty Clip coupler, but the relative attenuation over all 11 cadaveric temporal bones was $<10 \mathrm{~dB}$. Conclusions: The new $\mathrm{SH}$ coupler provides an alternative with more stable fixation when placement of the current $\mathrm{Vi}$ broplasty Clip coupler is limited due to insufficient space after canal-wall-down mastoidectomy, while still delivering effective stimuli to the cochlea.

(C) 2021 The Author(s)

Published by S. Karger AG, Basel

\section{Introduction}

The Vibrant Soundbridge (VSB; MED-EL Medical Electronics, Austria) is an active middle ear implant with a floating mass transducer (FMT) for patients with conductive and mixed hearing loss. Specifically, the VSB pro-

Birthe Warnholtz and Merlin Schär: first authors.
Jae Hoon Sim

Department of Otorhinolaryngology, Head and Neck Surgery

University Hospital Zurich

Frauenklinikstrasse 24, CH-8091 Zürich (Switzerland)

JaeHoon.Sim@usz.ch 
vides benefits to patients for whom conventional hearing aids do not work efficiently, such as patients with aural atresia [Frenzel et al., 2010; Barillari et al., 2012; Zernotti et al., 2013; McKinnon et al., 2014; Yang et al., 2014; Wang et al., 2016; Célérier et al., 2017; Leinung et al., 2017], recurrent or chronic otitis externa, eczema, ear canal irritations, external ear aplasia, extraordinarily narrow ear canals [Ihler et al., 2014a, 2014b], allergies to hearing aid molds, and chronic stenosing otitis externa [Thomas et al., 2017; Skarzynski et al., 2018]. The FMT of the VSB is attached to structures in the middle ear cavity, such as the incus [Eze et al., 2014; Schraven et al., 2014; Célérier et al., 2017], the stapes [Frenzel et al., 2010; Beleites et al., 2011; Park et al., 2014], and the round window [Luetje et al., 2002; Baumgartner et al., 2010; Barillari et al., 2012], using proper couplers. While the coupling location of the FMT is determined by the status of the middle ear structures, it is known that coupling of the FMT on the stapes head $(\mathrm{SH})$ delivers larger stimulus levels to the cochlea than coupling to other structures [Cremers et al., 2008; Chen et al., 2017]. Considering that the effective stimulus from the middle ear ossicular chain to the cochlea is mainly determined by the piston-like motion of the stapes [Eiber et al., 2012; Edom et al., 2013], it is presumed that coupling of the FMT to the SH is an efficient way to produce the piston-like motion of the stapes.

The Vibroplasty Clip coupler, consisting of 4 legs that couple to the stapes suprastructure [Hüttenbrink et al., 2011], is most widely used to couple the FMT to the SH. With this coupler, the FMT is vertically aligned above the $\mathrm{SH}$, and such a location and alignment of the FMT efficiently delivers stimuli to the cochlea. However, its use is sometimes limited due to insufficient space for the FMT, especially when the surgical opening is a canal-wall-down mastoidectomy. As an alternative to the current Vibroplasty Clip coupler, a new SH coupler (version 0.22, MED-EL Medical Electronics, Austria) has been developed. With the new SH coupler, the FMT is positioned on the inferior side of the stapes instead of the top of the $\mathrm{SH}$ to reduce the functional height. Further, the new SH coupler includes coupling to the stapes crura in addition to coupling to the SH to enhance stability of angular positioning of the FMT.

The research design for this study was a comparative analysis between the current Vibroplasty Clip coupler and the new SH coupler. Using cadaveric temporal bones, placement of the couplers with the FMT was examined, and expected surgical outcomes were assessed by measuring piston-like motion of the stapes footplate for the 2 couplers.

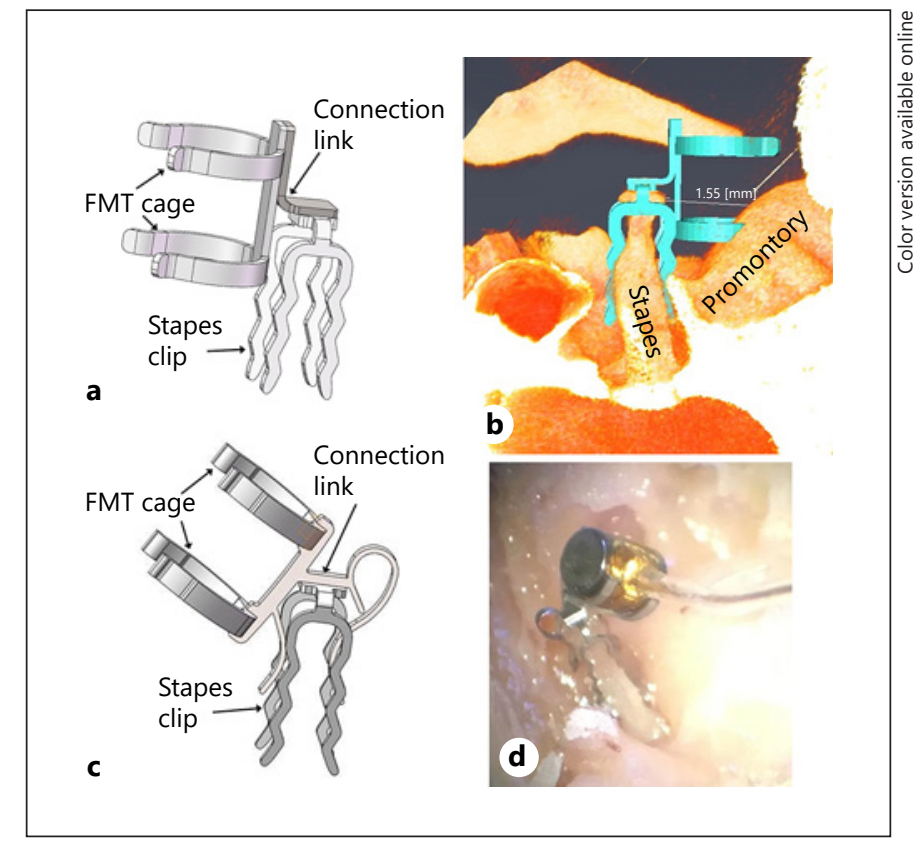

Fig. 1. New SH coupler and its placement. Initial design of the SH coupler (a) and its placement tested with $\mu-\mathrm{CT}$ data (b), and the upgraded version of the $\mathrm{SH}$ coupler (version $0.22 ; \mathbf{c}$ ) and its placement on the TB (d). SH, stapes head; $\mu$-CT, micro-computed tomography; FMT, floating mass transducer.

\section{Material and Methods}

Approval of this study was granted by the Swiss Ethic Commission of Canton Zürich with the identification number KEK-ZHNr. 2014-0544.

\section{New SH Coupler}

The new SH coupler consists of (1) a stapes clip, (2) the FMT cage, and (3) a connection link (Fig. 1a, c). The stapes clip provides legs for fastening the $\mathrm{SH}$ coupler to the crura of the stapes, and the FMT is attached to the FMT cage. The connection link provides a connection between the stapes clip and FMT cage and provides for coupling onto the SH as well. The connection link was initially designed such that the FMT is positioned on the inferior side of the stapes and is oriented parallel to the longitudinal direction (i.e., lateral-medial direction) of the stapes (Fig. 1a). However, with the initial design, space for placement of the FMT was insufficient and placement without touching the promontory was impossible. Such lack of space was confirmed by examination with digital simulation of the alignment via middle ear models based on micro-computed tomography $(\mu-\mathrm{CT})$ data. Six sets of $\mu$-CT data were used for the digital simulation, and the initial design could not be placed in 5 out of 6 sets. Figure $1 \mathrm{~b}$ shows the spatial interference of the promontory with the FMT cage. In order to allow space for the FMT, the SH coupler was redesigned, especially the connection link, which was bent such that the angle between the longitudinal direction of the FMT and the longitudinal direction of the stapes was $30^{\circ}$ in the new design (Fig. 1c). With 
the modified design, placement of the coupler was not impeded by the promontory in the digital simulation with the same $\mu$-CT data sets.

\section{Temporal Bone Preparation}

Cadaveric temporal bones (TBs) were obtained from Science Care (Phoenix, AZ, USA). Eleven TBs (excluding TBs that did not show normal middle ear characteristics; see section "Test of 'Normal' Temporal Bone Function") from 7 female and 4 male subjects were used in this study. The average age of the donors was 73.3 years (ranging from 43 to 87 years). The TBs were collected 48 $72 \mathrm{~h}$ after death and were immediately frozen by the provider. They were kept frozen at $-20^{\circ} \mathrm{C}$ until they were prepared for measurement.

The TBs were prepared for test fitting of the couplers according to the American Society for Testing and Materials (ASTM) standard [F2504-05, Philadelphia, 2005]. An extended mastoidectomy with posterior tympanotomy was performed to expose the middle ear ossicular chain [Gerig et al., 2015; Cuny et al., 2019]. The external ear canal was removed and replaced by an artificial ear canal made of a plastic tube (an inner diameter of $9 \mathrm{~mm}$ ). A loudspeaker (ER-2, Etymotic Research, Elk Grove Village, IL, USA) and microphone probe (ER-14C, Etymotic Research, Elk Grove Village, IL, USA) together with a yellow foam eartip were placed in the artificial ear canal; the distance between the microphone probe tip and the center of the tympanic membrane was approximately $3-$ $5 \mathrm{~mm}$.

After measurement for the test fitting to the ASTM standard, a section of the middle ear, including the tympanic membrane and malleus-incus complex, was removed. A portion of the bony wall was drilled away as well, for easy access of the coupler prosthesis and the laser beam from the laser Doppler vibrometer (LDV) systems. Care was taken to keep the stapedial tendon and the cochlear structures intact. Each specimen was periodically moistened during the measurements to prevent drying.

\section{Test of "Normal" Temporal Bone Function}

To determine whether the TBs exhibited normal function, physiological stapes motions in response to acoustic stimuli were examined and compared with the ASTM standard [F2504-05, Philadelphia, 2005]. Assessment of the stapes motion was done at $0.5,1,2,3$, and $4 \mathrm{kHz}$ because the averaged values across samples in the ASTM standard do not reflect normal performance at higher frequencies [Morse et al., 2019]. While it is known that the velocity curve of an individual stapes has a steep notch near $5-7 \mathrm{kHz}$ [e.g., Sim et al., 2010], the notch is obscured when the velocity curves are averaged from a large number of samples, as is the case in the ASTM standard. Physiological motions of the stapes were measured near the footplate center using a single-point LDV system (CLV-2534, Polytec GmbH, Germany). The TBs exhibiting performance beyond the range of "normal" (i.e., 95\% confidence interval) in the ASTM standard were excluded for further preparation and measurements. Figure 2 shows the stapes motion of 11 TBs in response to acoustic stimuli, all of which were within the ASTM standard and were used for this study.

\section{Coupler Placement}

Placement of the Vibroplasty Clip coupler and the new SH coupler onto the stapes was examined for the $11 \mathrm{TBs}$ with the following objectives: (1) neither the FMT nor the coupler could touch the

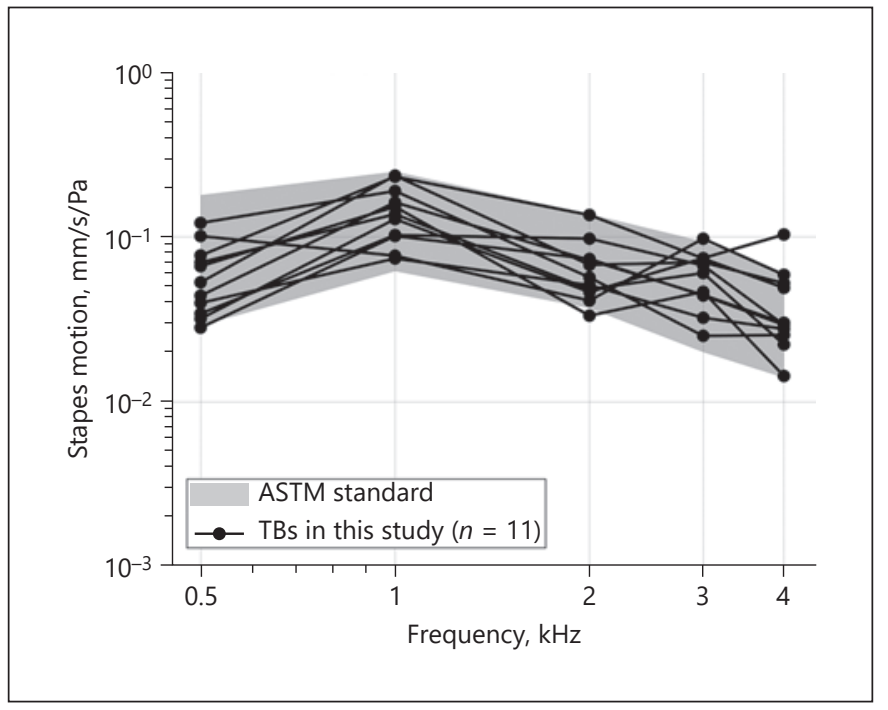

Fig. 2. Physiological motion of the stapes in response to acoustic stimuli, in comparison with the ASTM standard. ASTM, American Society for Testing and Materials; TBs, temporal bones.

promontory or its peripheral bones, (2) the coupling could be made such that the position of the coupler was stable, and (3) the coupler could be placed onto the stapes without a gap between the plate of the connection link and the SH. When all 3 conditions were satisfied, the placement was considered "perfect." If the first and second conditions were satisfied but the third condition was not, the placement was considered "imperfect but stable." If either the first or second condition could not be satisfied, the placement was considered a "failure."

\section{Measurement of Effective Stimuli to the Cochlea}

The stapes footplate velocities excited by the FMT via the new $\mathrm{SH}$ coupler were measured and compared with the corresponding velocities via the current Vibroplasty Clip coupler. Harmonic signals of 900 frequencies distributed logarithmically between 0.4 and $10 \mathrm{kHz}$ were generated by an audio analyzer (APx585, Audio Precision Inc., Beaverton, OR, USA) and were delivered to the FMT via an audio amplifier (RMX 850a, QSC Audio Products, Costa Mesa, CA, USA). The sampling rate was set at $192 \mathrm{kS} / \mathrm{s}$. The resistance of the FMT was measured by an Ohmmeter, and the voltage to the FMT was set such that the current on the FMT was $1 \mathrm{~mA}$ root-mean-square. Motion of the stapes footplate was measured near the footplate center, where the motion is presumed to be similar to the piston-like component of the stapes [Sim et al., 2010; Lauxmann et al., 2012], using a Short Wave Infrared LDV system (Scan-Sense, OptoMET GmbH, Germany). The direction of the laser beam was tilted by approximately $20-30^{\circ}$ superiorly relative to the direction normal to the footplate.

A post hoc analysis with paired $t$ tests was done to compare the Vibroplasty Clip coupler and the new SH coupler. The post hoc analysis was performed for 8 frequency bands $(0.4-0.75,0.75-1$, $1-1.5,1.5-2,2-3,3-4,4-6$, and $6-8 \mathrm{kHz})$. In each frequency band, measurement results were averaged. The difference was considered to be statistically significant for $p$ values $<0.05$. 


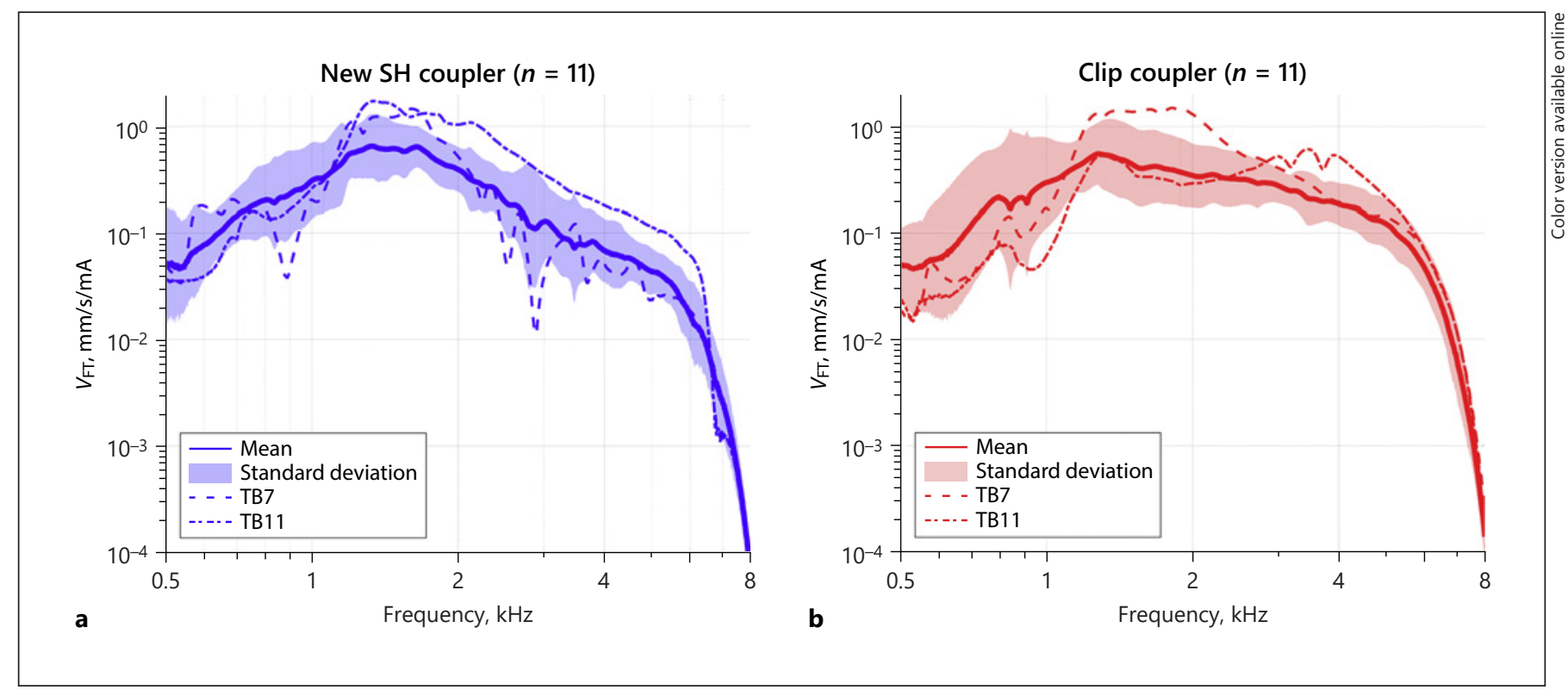

Fig. 3. Velocities of the stapes footplate center per current on the FMT (1 mA) in 11 TBs, with the SH coupler (a) and the Vibroplasty Clip coupler (b). FMT, floating mass transducer; SH, stapes head; TB, temporal bone.

Table 1. Cases of “imperfect but stable” placements

\begin{tabular}{lll}
\hline & New SH coupler & Vibroplasty Clip coupler \\
\hline TB7 & Gap between the SH and the plate of the connection & "Perfect" placement \\
\hline TB8 & "Perfect" placement & Legs on the inferior side were cut \\
\hline TB11 & Gap between the SH and the plate of the connection & Gap between the SH and the plate of the FMT cage \\
\hline \multicolumn{2}{c}{ SH, stapes head; TB, temporal bone. }
\end{tabular}

\section{Results}

\section{Coupler Placement}

Placing the coupler on the stapes could be done without any problem (i.e., "perfect" placement in section "Coupler Placement") in 9 of 11 temporal bones for both couplers. A "failure" (see section "Coupler Placement") was not observed for either of the 2 couplers. Placement of the coupler was not considered a "failure" but was partially problematic (i.e., "imperfect but stable" in section "Coupler Placement") in TB7 (81 years, female) and TB11 (78 years, female) for the new SH coupler and in TB8 (71 years, male) and TB11 for the Vibroplasty Clip coupler. In TB7 and TB11, a small gap (estimated as $\sim 0.1$ $\mathrm{mm}$, not measured) between the $\mathrm{SH}$ and the plate of the connection link was introduced to prevent the FMT for the new SH coupler from touching the promontory. In TB8, the legs of the Vibroplasty Clip coupler on the inferior side had to be cut to avoid touching the promontory. In TB11, a small gap between the $\mathrm{SH}$ and the plate of the FMT cage of the Vibroplasty Clip coupler was unavoidable due to a relatively large $\mathrm{SH}$. All the cases of "imperfect but stable" placements for the new SH coupler and the Vibroplasty Clip coupler are summarized in Table 1.

\section{Relative Attenuation of Effective Stimuli to the}

Cochlea with the New SH Coupler

Figure 3 shows velocities at the footplate center with respect to the current on the FMT (per $1 \mathrm{~mA}$ ) for 11 temporal bones. Generally, velocities at the footplate center show similar magnitudes for the Vibroplasty Clip coupler 
Fig. 4. Relative magnitude ratio of the stapes velocities between the new $\mathrm{SH}$ coupler and the Vibroplasty Clip coupler (mean and standard deviation). A significant difference $(p<0.05)$ between the 2 couplers was observed at frequencies above $3 \mathrm{kHz}$ (shaded with dark gray in the figure). $\mathrm{SH}$, stapes head.

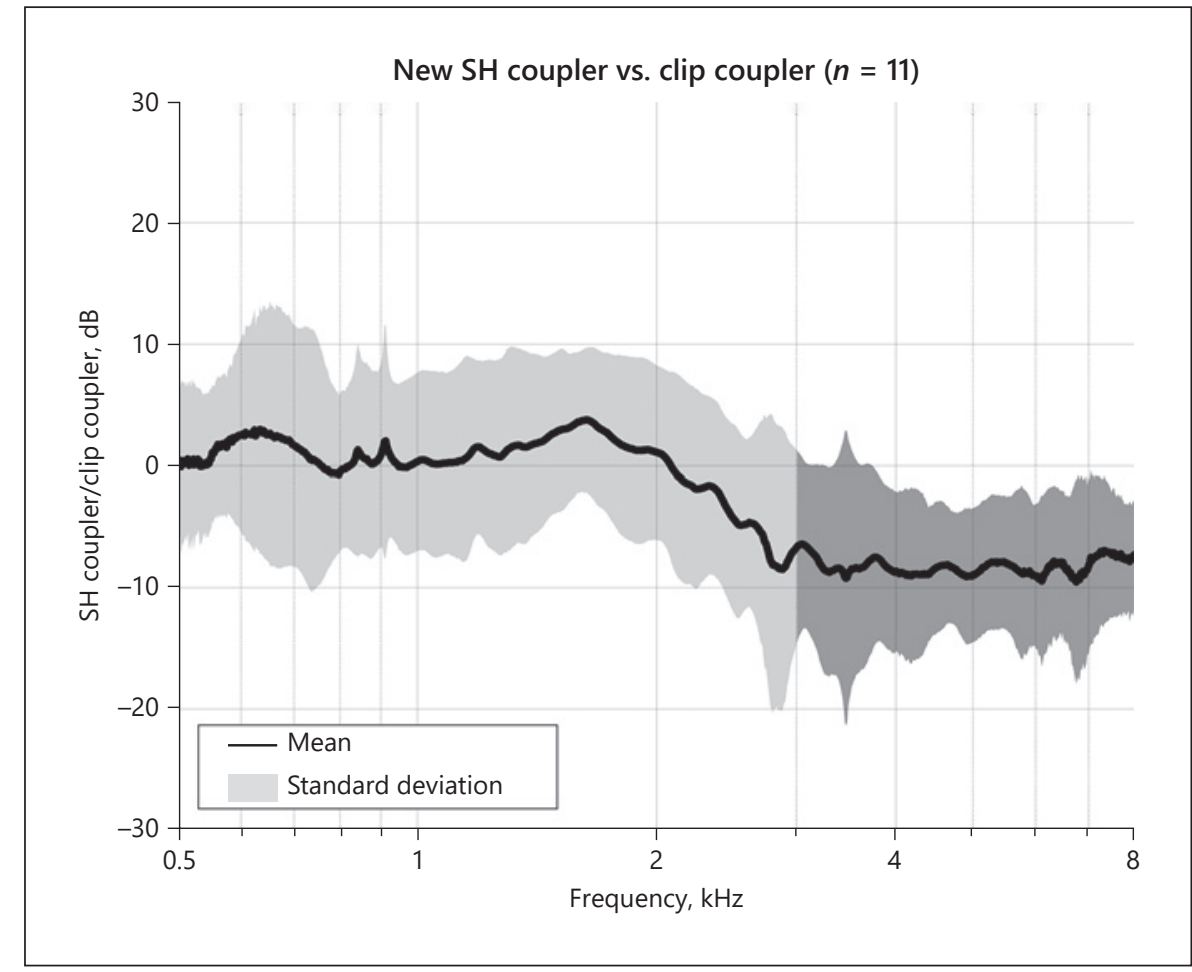

Fig. 5. Functional height of the FMT $\left(h_{f}\right)$ in a canal-wall-down mastoidectomy (sketch), with the new SH coupler (a, $1.9 \mathrm{~mm})$ and the Vibroplasty Clip coupler (b, $2.4 \mathrm{~mm}$ ). FMT, floating mass transducer; $\mathrm{SH}$, stapes head.

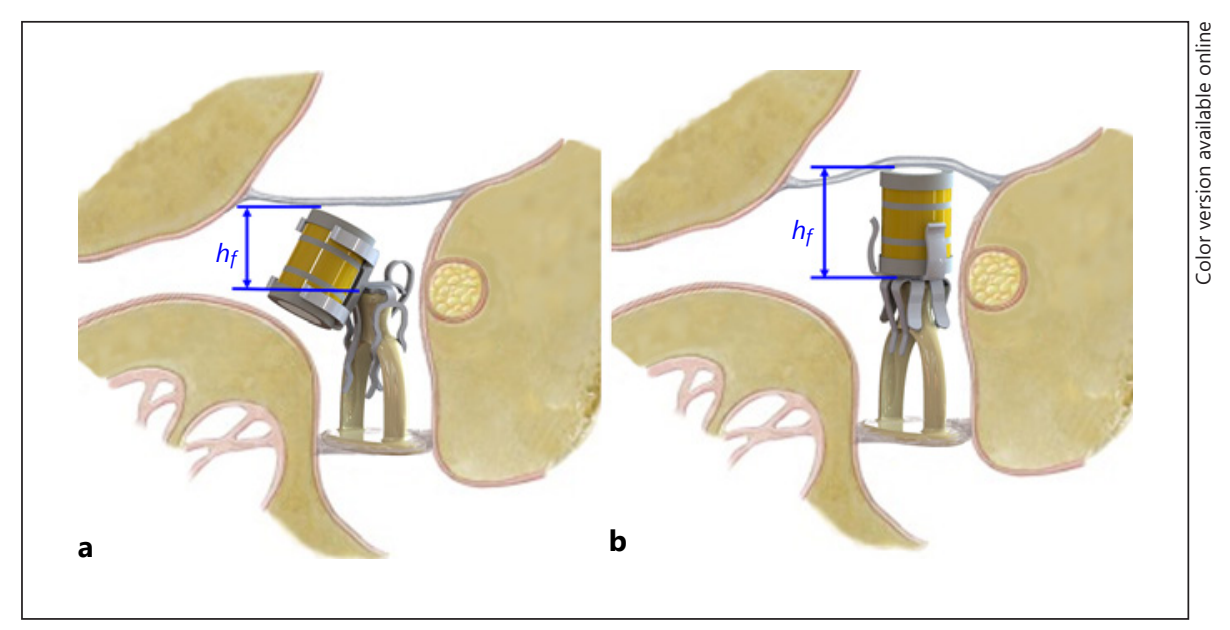

and the new $\mathrm{SH}$ coupler up to $2 \mathrm{kHz}$ and lower magnitudes for the $\mathrm{SH}$ coupler above $3 \mathrm{kHz}$.

For comparison of effective stimuli to the cochlea between the 2 couplers, the magnitude ratios of the velocities with the new SH coupler relative to the velocities with the Vibroplasty Clip coupler were calculated, and the means and standard deviations of the relative magnitude ratios are shown in Figure 4. The relative magnitude ratio was calculated for each $\mathrm{TB}$, and relative ratios were averaged over the $11 \mathrm{TBs}$. The difference was $<10 \mathrm{~dB}$ on aver- age up to $8 \mathrm{kHz}$. Post hoc analysis of frequency bands showed statistically significant differences between the Vibroplasty Clip coupler and the SH coupler for frequency bands above $3 \mathrm{kHz}$ ( $p=0.0049$ for $3-4 \mathrm{kHz}, p=0.0001$ for $4-6 \mathrm{kHz}$, and $p=0.0005$ for $6-8 \mathrm{kHz}$ ). Frequency bands with statistically significant differences are shaded with dark gray in Figure 4. Statistically significant differences were not observed for frequency bands below $3 \mathrm{kHz}$ $(p=0.1352-0.9115)$. 


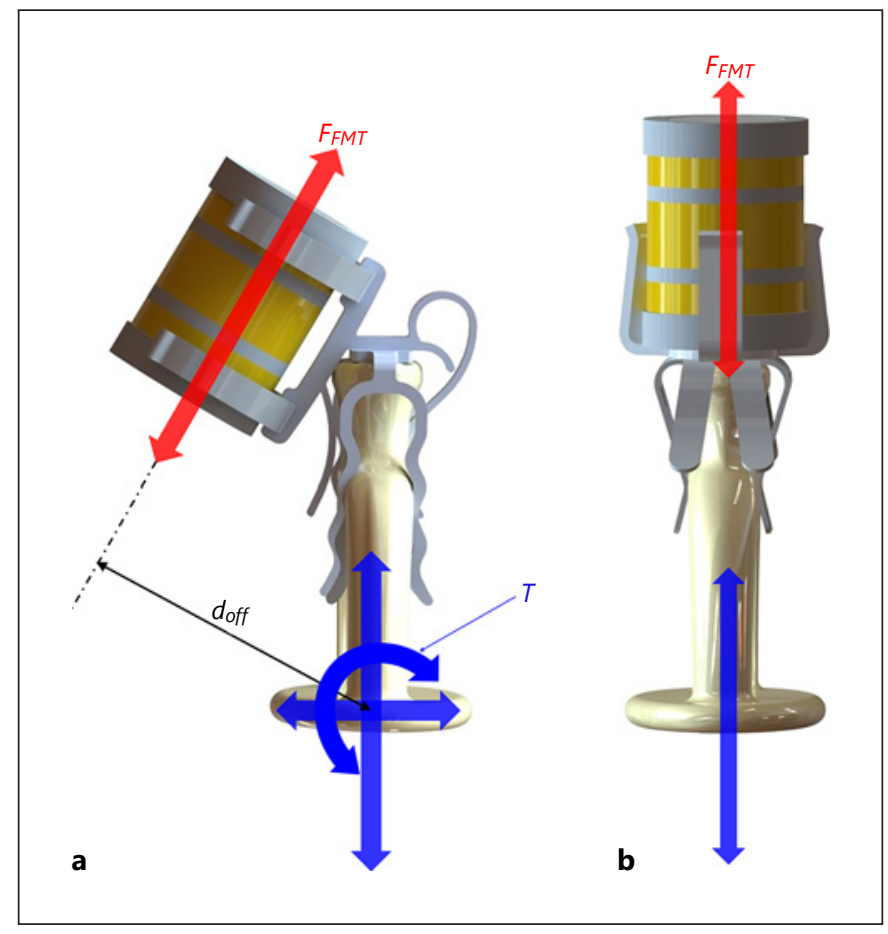

Fig. 6. Stimulation force by the FMT ( $F_{\mathrm{FMT}}$, red in the figure) and its equivalent representation with respect to the center of the stapes footplate (blue in the figure), with the new SH coupler (a) and the Vibroplasty Clip coupler (b). It was assumed that the stimulation force is along its longitudinal direction. A torque $T\left(=d_{\mathrm{off}} / F_{\mathrm{FMT}}\right)$ on the center of the stapes footplate due to a distance $d_{\text {off }}$ between its longitudinal direction pf the FMT and the center of stapes footplate. FMT, floating mass transducer; SH, stapes head.

The small gap between the $\mathrm{SH}$ and the plate of the connection link for placement of the SH coupler (see section "Coupler Placement") was presumed to have negligible effect on stapes velocity. In TB7 and TB11, where a small gap was introduced for placement of the $\mathrm{SH}$ coupler, substantial attenuation of the stapes velocity was not observed (Fig. 3).

\section{Discussion}

The new coupler used in this study was developed to reduce the functional height of the FMT, which is defined as the distance from the top of $\mathrm{SH}$ to the top edge of the FMT $\left(h_{\mathrm{f}}\right.$ in Fig. 5). While the functional height with the current Vibroplasty Clip coupler is $2.4 \mathrm{~mm}$, it is $1.9 \mathrm{~mm}$ for the new SH coupler. The functional height of the Vibroplasty Clip coupler may not be problematic in cases that the previous surgery was canal-wall-up mastoidec- tomy. However, during canal-wall-down mastoidectomy, the posterior and superior canal walls are drilled down to the level of the facial nerve in its mastoidal segment, resulting in a reduced volume of the middle ear space [Whittemore et al., 1998]. This is also the case in atelectatic ears or if the malleus is medialized. In these situations, when the stapes superstructure is intact but the middle ear cleft is shallow, the new coupler will be useful because of its reduced functional height. Further, treatment with the VSB is presumed to be a good option for patients with radical cavities because the ossiculoplasty and conventional hearing aids are inappropriate for most of these patients [Ihler et al., 2014].

With respect to the stability of the attachment between the coupler and stapes, the 4 legs of the Vibroplasty Clip coupler contact a part near the SH and consequently may not provide strong fixation against rotational dislocation of the FMT. However, the new SH coupler is fixed on the crura as well as the head of the stapes and therefore provides firm fixation against rotational dislocation of the FMT. With the initial design of the new SH coupler (Fig. 1a), placement of the SH coupler was spatially impeded by the promontory. However, the issue could be resolved by modifying the design of the connection link of the coupler such that the angle between the longitudinal direction of the FMT and the longitudinal direction of the stapes was $30^{\circ}$ (Fig. 1c). "Failure" in placement of the $\mathrm{SH}$ coupler was not observed with the new design (section "Coupler Placement").

The piston-like motion of the stapes footplate was measured to assess the effective stimulus to the cochlea in this study, and relative attenuation of the effective stimulus above $3 \mathrm{kHz}$ with the new $\mathrm{SH}$ coupler is presumed to be due to a relatively large rocking-like motion of the stapes footplate. The relatively large rocking-like motion with the $\mathrm{SH}$ coupler can be caused by 2 possible mechanism. First, location and orientation of the FMT with new SH coupler generate a torque with respect to the center of the mass of the stapes footplate. Figure 6 illustrates the stimulation force by the FMT $\left(F_{\mathrm{FMT}}\right.$, red in the figure) and equivalent representation of the stimulation force with respect to the center of the stapes footplate (blue in the figure), assuming that the stimulation force is along its longitudinal direction. The longitudinal direction of the FMT with the new SH coupler has a distance $d_{\text {off }}$ from the center of the stapes footplate, which is due to the offset and tilting of the FMT, and thus a torque $T\left(=d_{\text {off }} / F_{\mathrm{FMT}}\right)$ is generated. In the case that the FMT is mounted with the Vibroplasty Clip coupler, the longitudinal direction of the FMT passes through the center of 
the stapes footplate (i.e., $d_{\text {off }}=0$ ), and thus no torque about the center of the stapes footplate is generated. Eiber et al. [2012] showed that a large rocking-like motion of the stapes footplate is generated when stimulation on the $\mathrm{SH}$ is not perpendicular to the footplate, and thus a torque with respect to the center of the stapes footplate is generated. It was also shown that a larger piston-like motion is generated when the longitudinal direction of the FMT on the $\mathrm{SH}$ is parallel to the stapes footplate and thus has a distance (i.e., $d_{\text {off }} \neq 0$ ) from the center of the stapes footplate [Sim et al., 2016; Chatzimichalis et al., 2018; Dobrev et al., 2018]. Second, in addition to the torque $T$, the center of mass of the structure moves toward the inferior side when the FMT is mounted with the SH coupler, due to the biased position of the FMT. The FMT is positioned approximately on the center line of the stapes when the FMT is mounted with the Vibroplasty Clip coupler. According to one theory of dynamics, such a nonsymmetrical mass distribution is expected to generate rocking-like motion of the stapes, especially at high frequencies above the resonance (mass-dominant region). Considering the result of this study that relative attenuation of the effective stimulus with the new SH coupler was observed above $3 \mathrm{kHz}$, the bias of the center of the mass with the $\mathrm{SH}$ coupler may have more dominant effects.

Attenuation of the effective stimuli with the SH coupler relative to the effective stimuli with the Vibroplasty Clip coupler was observed only at high frequencies above $3 \mathrm{kHz}$ with the amount of $<10 \mathrm{~dB}$ (Fig. 4). From the point of the effective stimuli, using the Vibroplasty Clip coupler is a better option than use of the new SH coupler if application of the Vibroplasty Clip coupler is possible. However, when the Vibroplasty Clip coupler cannot be placed due to insufficient middle ear space, the new $\mathrm{SH}$ coupler can be a good alternative only with a small amount of attenuation at high frequencies. Previous studies [Cremers et al., 2008; Chen et al., 2017] showed relatively large stapes motion at high frequencies when the FMT was coupled onto the SH compared to stapes motion when the FMT was coupled to the long process of the incus, which is the most typical position for the FMT coupler. Chen et al. [2017] showed that coupling of the FMT to the SH using the Vibroplasty Clip coupler produced approximately $20 \mathrm{~dB}$ higher stapes motion above $3 \mathrm{kHz}$ than coupling to the long process of the incus. Therefore, coupling using the new SH coupler with the relative attenuation of $<10 \mathrm{~dB}$ is expected to show a better sound transmission than typical coupling of the FMT to the long process of the incus.

\section{Conclusion}

The new design of the SH coupler (Version 0.22) was assessed in comparison with the current Vibroplasty Clip coupler. The assessment focused on (1) placement of the coupler onto the stapes and (2) measurement of effective stimuli to the cochlea.

The new SH coupler provided a stable and firm fixation to the stapes with coupling to the stapes crura in addition to the SH. With the new SH coupler, a small gap between the $\mathrm{SH}$ and the plate of the connection link part was sometimes ( 2 out of 11 cases in this study) unavoidable in order to prevent the FMT from touching the promontory. However, according to the results of this study, effective stimuli to the cochlea, which were assessed by measuring velocities of the stapes footplate, were presumed not to be attenuated considerably, even with the gap (Fig. 3).

The effective stimuli to the cochlea with the new $\mathrm{SH}$ coupler were reduced at frequencies above $3 \mathrm{kHz}$ compared to the effective stimuli with the current Vibroplasty Clip coupler. However, relative attenuation was $<10 \mathrm{~dB}$ and therefore is not considered significant.

The new SH coupler provides efficient use of space in the middle ear cavity by moving position of the FMT to the inferior side of the stapes instead of positioning it above the SH (i.e., lateral side of the stapes) as with the Vibroplasty Clip coupler. Considering the results, the SH coupler introduced in this study provides an alternative, with more stable fixation, when placement of the current Vibroplasty Clip coupler is limited due to insufficient middle ear depth such as after canal-wall-down mastoidectomy and middle ear atelectasis, while still delivering effective stimuli to the cochlea.

\section{Statement of Ethics}

This study was executed under the ethical approval by the Swiss Ethic Commission of Canton Zürich with the identification number KEK-ZH-Nr. 2014-0544.

\section{Conflict of Interest Statement}

Kathrin Sonntag, an author of this manuscript, has been employed by MED-EL Medical Electronics (Austria), the manufacturer of the Vibroplasty Clip coupler and the new SH coupler in this manuscript. Otherwise, the authors have no conflicts of interest to declare. 


\section{Funding Sources}

This work has been financially supported by MED-EL Medical Electronics (Austria).

\section{Author Contributions}

All authors have been involved in the design of the study, performing experiments, data analysis, and/or writing the manuscript and have read and concur with the content of the manuscript.

\section{References}

American Society for Testing and Materials (ASTM). F2504-05 (2005). Standard practice for describing system output of implantable middle ear hearing devices. Philadelphia, PA; 2005.

Barillari M, Cerini R, Carner M, Cacciatori C, Spagnolli F, Cardobi N, et al. Congenital aural atresia treated with floating mass transducer on the round window: 5 years of imaging experience. Radiol Med. 2012;117(3):488-99.

Baumgartner WD, Böheim K, Hagen R, Müller J, Lenarz T, Reiss $S$, et al. The vibrant soundbridge for conductive and mixed hearing losses: European multicenter study results. Adv Otorhinolaryngol. 2010;69:38-50.

Beleites T, Neudert M, Beutner D, Hüttenbrink $\mathrm{KB}$, Zahnert T. Experience with vibroplasty couplers at the stapes head and footplate. Otol Neurotol. 2011;32(9):1468-72.

Célérier C, Thierry B, Coudert C, Blanchard M, Loundon N, Garabédian EN, et al. Results of VSB implantation at the short process of the incus in children with ear atresia. Int J Pediatr Otorhinolaryngol. 2017;93:83-7.

Chatzimichalis M, Dobrev I, Schär M, Röösli C, Sim JH. Cochlear activation by spatial motion of the stapes: Measurement of intracochlear pressure change. British Academic Conference in Otolaryngology (BACO); $2018 \mathrm{Jul}$ 4-6; Manchester, UK; 2018.

Chen T, Ren LJ, Yin DM, Li J, Yang L, Dai PD, et al. A comparative study of MED-EL FMT attachment to the long process of the incus in intact middle ears and its attachment to disarticulated stapes head. Hear Res. 2017;353:97103.

Cremers CW, Verhaegen VJ, Snik AF. The floating mass transducer of the vibrant soundbridge interposed between the stapes and tympanic membrane after incus necrosis. Otol Neurotol. 2008;30(1):76-8.

Cuny P, Alsolami NJ, Dobrev I, Warnholtz B, Röösli C, Sim JH. Influence of angular positioning of the prosthesis in stapes surgeries with a NiTiBond prosthesis: investigation in cadaveric temporal bones. Hear Res. 2019; 378:149-56.

Dobrev I, Schär M, Pfiffner F, Prochazka L, Röösli C, Huber A, et al. A comprehensive biomechanical model of the human stapes with 3D cochlear input impedance. The 39th Association for Research in Otolaryngology MidWinter Meeting; San Diego, CA; 2018.

Edom E, Obrist D, Henniger R, Kleiser L, Sim JH, Huber AM. The effect of rocking stapes motions on the cochlear fluid flow and on the basilar membrane motion. J Acoust Soc Am. 2013;134(5):3749-58.
Eiber A, Huber AM, Lauxmann M, Chatzimichalis M, Sequeira D, Sim JH. Contribution of complex stapes motion to cochlea activation. Hear Res. 2012;284(1-2):82-92.

Eze N, Mirón A, Rogers G, Jeronimidis G, O'Connor AF, Jiang D. The effect of angulation of the vibrating floating mass transducer on stapes velocity. Otol Neurotol. 2014;35(7): 1223-7.

Frenzel H, Hanke F, Beltrame M, Wollenberg B. Application of the vibrant soundbridge in bilateral congenital atresia in toddlers. Acta Otolaryngol. 2010;130(8):966-70.

Gerig R, Ihrle S, Röösli C, Dalbert A, Dobrev I, Pfiffner F, et al. Contribution of the incudomalleolar joint to middle-ear sound transmission. Hear Res. 2015;327:218-26.

Hüttenbrink KB, Beutner D, Bornitz M, Luers JC, Zahnert T. Clip vibroplasty: experimental evaluation and first clinical results. Otol Neurotol. 2011;32(4):650-3.

Ihler F, Bewarder J, Blum J, Matthias C, Canis M. Long-term functional outcome and satisfaction of patients with an active middle ear implant for sensorineural hearing loss compared to a matched population with conventional hearing aids. Eur Arch Otorhinolaryngol. 2014a;271(12):3161-9.

Ihler F, Köhler S, Meyer AC, Blum J, Strenzke N, Matthias C, et al. Mastoid cavity obliteration and vibrant soundbridge implantation for patients with mixed hearing loss. Laryngoscope. 2014;124(2):531-7.

Ihler F, Köhler S, Meyer AC, Blum J, Strenzke N, Matthias C, et al. Mastoid cavity obliteration and vibrant soundbridge implantation for patients with mixed hearing loss. Laryngoscope. 2014b;124(2):531-7.

Lauxmann M, Eiber A, Heckeler C, Ihrle S, Chatzimichalis M, Huber A, et al. In-plane motions of the stapes in human ears. J Acoust Soc Am. 2012;132(5):3280-91.

Leinung M, Zaretsky E, Lange BP, Hoffmann V, Stöver T, Hey C. Vibrant soundbridge in preschool children with unilateral aural atresia: acceptance and benefit. Eur Arch Otorhinolaryngol. 2017;274:159-65.

Luetje CM, Brackman D, Balkany TJ, Maw J, Baker RS, Kelsall D, et al. Phase III clinical trial results with the vibrant soundbridge implantable middle ear hearing device: a prospective controlled multicenter study. Otolaryngol Head Neck Surg. 2002;126(2):97-107.

McKinnon BJ, Dumon T, Hagen R, Lesinskas E, Mlynski R, Profant M, et al. Vibrant soundbridge in aural atresia: does severity matter? Eur Arch Otorhinolaryngol. 2014;271(7): 1917-21.
Morse RP, Mitchell-Innes A, Prokopiou AN, Irving $\mathrm{RM}$, Begg PA. Inappropriate use of the "rosowski criteria" and "modified rosowski criteria" for assessing the normal function of human temporal bones. Audiol Neurotol. 2019;24(1):20-4.

Park AY, Jeon JH, Moon IS, Choi JY. A case of the vibrant soundbridge stapes coupler in patients with mixed hearing loss. Korean $\mathrm{J}$ Audiol. 2014;18(2):93-6.

Schraven SP, Dalhoff E, Wildenstein D, Hagen R, Gummer AW, Mlynski R. Alternative fixation of an active middle ear implant at the short incus process. Audiol Neurootol. 2014;19(1): $1-11$.

Sim JH, Chatzimichalis M, Lauxmann M, Röösli C, Eiber A, Huber AM. Complex stapes motions in human ears. J Assoc Res Otolaryngol. 2010;11(3):329-41.

Sim JH, Pfiffner F, Prochazka L, Dobrev I, Péus D, Dalbert A, et al. Effective stimulus to the cochlea: intracochlear pressure measurement. In: IUTAM symposium on advances in biomechanics of hearing. Stuttgart, Germany: International Union of Theoretical and Applied Mechanics; 2016 May 17-20.

Skarzynski PH, Osinska K, Krol B, Wawszczyk S, Matusiak M, Ratuszniak A, et al. Use of the vibrant soundbridge middle ear implant with short process incus coupler for chronic obstructive inflammation of the external ear canal: case study. J Hear Sci. 2018;8(2):25-31.

Thomas JP, Voelter C, Neumann K, Dazert S. Vibroplasty in severe congenital or acquired meatal stenosis by coupling an active middle ear implant to the short process of the incus. Otol Neurotol. 2017;38(7):996-1004.

Wang D, Zhao S, Zhang Q, Li Y, Ma X, Ren R. Vibrant soundbridge combined with auricle reconstruction for bilateral congenital aural atresia. Int J Pediatr Otorhinolaryngol. 2016; $86: 240-5$.

Whittemore KR Jr, Merchant SN, Rosowski JJ. Acoustic mechanisms: canal wall-up versus canal wall-down mastoidectomy. Otolaryngol Head Neck Surg. 1998;118(6):751-61.

Yang SM, Zou YH, Li JN, Jiao QS, Yi HJ, Han DY. Vibrant soundbridge implantation via the third window in two Chinese patients with severe bilateral congenital aural atresia. Acta Otolaryngol. 2014;134(1):1-6.

Zernotti ME, Arauz SL, Di Gregorio MF, Arauz SA, Tabernero P, Romero MC. Vibrant soundbridge in congenital osseous atresia: multicenter study of 12 patients with osseous atresia. Acta Otolaryngol. 2013;133(6):56973.

Warnholtz/Schär/Cuny/Sonntag/Beutner/ Dobrev/Röösli/Sim 